\title{
Cortical Correlates of the Auditory Frequency-Following and Onset Responses: EEG and fMRI Evidence
}

\author{
탈 Emily B.J. Coffey, ${ }^{1,2,3}$ @Gabriella Musacchia, ${ }^{4}$ and Robert J. Zatorre ${ }^{1,2,3}$ \\ ${ }^{1}$ Montreal Neurological Institute, McGill University, Montréal, Québec, Canada H3A 2B4, ${ }^{2}$ International Laboratory for Brain, Music, and Sound Research \\ (BRAMS), Montreal, Québec, Canada, ${ }^{3}$ Centre for Research on Brain, Language and Music (CRBLM), Montréal, Québec, Canada H3G 2A8, and \\ ${ }^{4}$ Department of Speech-Language Pathology and Audiology, University of the Pacific, Stockton, California 95207
}

The frequency-following response (FFR) is a measure of the brain's periodic sound encoding. It is of increasing importance for studying the human auditory nervous system due to numerous associations with auditory cognition and dysfunction. Although the FFR is widely interpreted as originating from brainstem nuclei, a recent study using MEG suggested that there is also a right-lateralized contribution from the auditory cortex at the fundamental frequency (Coffey et al., 2016b). Our objectives in the present work were to validate and better localize this result using a completely different neuroimaging modality and to document the relationships between the FFR, the onset response, and cortical activity. Using a combination of EEG, fMRI, and diffusion-weighted imaging, we show that activity in the right auditory cortex is related to individual differences in FFR-fundamental frequency $\left(f_{0}\right)$ strength, a finding that was replicated with two independent stimulus sets, with and without acoustic energy at the fundamental frequency. We demonstrate a dissociation between this FFR- $f_{0}$-sensitive response in the right and an area in left auditory cortex that is sensitive to individual differences in the timing of initial response to sound onset. Relationships to timing and their lateralization are supported by parallels in the microstructure of the underlying white matter, implicating a mechanism involving neural conduction efficiency. These data confirm that the FFR has a cortical contribution and suggest ways in which auditory neuroscience may be advanced by connecting early sound representation to measures of higher-level sound processing and cognitive function.

Key words: auditory cognition; EEG; fMRI; frequency-following response; onset response; spectrotemporal processing

\section{Significance Statement}

The frequency-following response (FFR) is an EEG signal that is used to explore how the auditory system encodes temporal regularities in sound and is related to differences in auditory function between individuals. It is known that brainstem nuclei contribute to the FFR, but recent findings of an additional cortical source are more controversial. Here, we use fMRI to validate and extend the prediction from MEG data of a right auditory cortex contribution to the FFR. We also demonstrate a dissociation between FFR-related cortical activity from that related to the latency of the response to sound onset, which is found in left auditory cortex. The findings provide a clearer picture of cortical processes for analysis of sound features.

\section{Introduction}

The frequency-following response (FFR) is an auditory signal recorded using EEG that offers a noninvasive view of behaviorally and clinically relevant individual differences in early sound processing (Krishnan, 2007; Skoe and Kraus, 2010; Kraus and WhiteSchwoch, 2015). Although the FFR itself is widely interpreted as having subcortical sources (Chandrasekaran and Kraus, 2010), its strength is correlated with measures of cortical waves (Musacchia et al., 2008) and is known to be modulated by cortical processes such as learning (Musacchia et al., 2007; Krishnan et al., 2009) and perhaps attention (Galbraith and Arroyo, 1993; Lehmann and Schönwiesner, 2014). Recent MEG evidence suggests that, in addition to generators in brainstem nuclei, there is a direct contribution from the auditory cortex at the fundamental 
frequency $\left(f_{0}\right)$ with a rightward bias (Coffey et al., 2016b). However, MEG localization is indirect, relying on distributed source modeling to localize and separate cortical from subcortical sources, an approach for which limitations are still being explored (Attal and Schwartz, 2013). Validation of cortical involvement using more direct complementary methods is thus essential.

Features of the FFR vary between people, even within a neurologically normal young adult population (Hoormann et al., 1992; Ruggles et al., 2012; Coffey et al., 2016a). These differences have been linked to musical (Musacchia et al., 2007; Strait et al., 2009; Bidelman, 2013) and language (Wong et al., 2007) experience and have been shown to be cognitively and behaviorally relevant, for example, in the perception of speech in noise (Ruggles et al., 2012), consonance and dissonance (Bones et al., 2014), and in pitch perception bias (Coffey et al., 2016a). Similarly, the MEG FFR- $f_{0}$ signal attributed to the right auditory cortex in our prior study was correlated with musical experience and fine frequency (FF) discrimination ability (Coffey et al., 2016b). These interindividual variations provide a means of testing the hypothesis of an FFR- $f_{0}$ contributor in the auditory cortex via fMRI: if stronger FFR- $f_{0}$ encoding is partly indicative of greater phase-locked neuronal activity in the right auditory cortex, then FFR $f_{0}$ strength should be positively correlated with the magnitude of the BOLD response in the same area due to the increased metabolic requirements of this neural population (Magri et al., 2012). A related question concerns the generalizability of the MEG findings to other sounds. Our prior MEG finding relied on a synthetic speech syllable that produces a clear, consistent onset response and FFR (Johnson et al., 2005; Skoe and Kraus, 2010). But the auditory system must also contend with sounds that include degraded or missing frequency information; to this end we used both the speech syllable and a piano tone

As well as identifying FFR- $f_{0}$-sensitive regions in the auditory cortex, it is useful to know if they can be dissociated from areas sensitive to other measures of early sound encoding, such as the timing of the transient onset response to sound, as suggested by behavioral dissociations (Johnson et al., 2005; Kraus and Nicol, 2005; Skoe and Kraus, 2010). If this is the case, we would expect measures of the onset response and the FFR to correlate with BOLD activity in different cortical populations. To clarify timing-related results, we also obtained measures of white matter microstructure, which are related to signal transmission speed (Wozniak and Lim, 2006).

In the present study, we measured neural responses to two periodic sounds using EEG and fMRI, and assessed the relationships between measures of FFR- $f_{0}$ strength, onset latency, and fMRI activity. Our primary aim was to test the hypothesis that individual differences in FFR- $f_{0}$ strength is correlated with the magnitude of fMRI response in the right auditory cortex. We tested three additional hypotheses: (1) that the FFR- $f_{0}$ BOLD relationship is robust to stimuli with and without a fundamental; (2) that an FFR- $f_{0}$-sensitive area can be dissociated from an onset latency-sensitive area; and (3) that timing-related results are correlated with the structure of the white matter directly underlying the auditory cortex.

\section{Materials and Methods}

Participants. We recruited 26 right-handed young adults divided into 2 groups: musicians who practiced at least 1 instrument regularly $(>1.5 \mathrm{~h}$ per week) and nonmusicians with minimal exposure to musical training. All subjects reported having normal hearing and no neurological conditions and were compensated for their time. Normal or corrected-tonormal vision (Snellen Eye Chart) and pure-tone thresholds from 250 to
$16 \mathrm{kHz}$ were measured to confirm sensory function (all but one subject had $\leq 20 \mathrm{~dB}$ HL pure-tone thresholds within the lower frequencies applicable to this study, $250-2000 \mathrm{~Hz}$; this subject was included as stimuli are presented well above threshold binaurally and the opposite ear had a normal threshold). One subject was excluded due to a technical problem. The remaining 25 subjects (mean age: 25.8, SD: 5.0, 13 females) included 13 musicians and 12 nonmusicians. Groups did not differ significantly in age (musicians mean: $25.2, \mathrm{SD}=5.6$; nonmusicians mean: $26.4, \mathrm{SD}=$ 4.5; Wilcoxon rank-sum test, two-tailed: $Z=0.84, p=0.40)$ or sex (7 musicians and 6 nonmusicians were female; $\chi^{2}$, two-tailed: $\chi_{(1,25)}^{2}=$ $0.04, p=0.85)$. Data about musical history were collected via an online survey (Montreal Music History Questionnaire, MMHQ; Coffey et al., 2011). Musicians reported an average of 10,300 h (SD: 5000) of vocal and instrumental practice and training; two nonmusicians reported $\sim 400 \mathrm{~h}$ of clarinet training as part of a school program; all others had no experience. The musicians varied in their instrument and musical style (main instruments: three keyboard, two woodwind, nine strings including five guitar; main styles: eight classical, four pop/rock, one traditional/folk). All experimental procedures were approved by the Montreal Neurological Institute Research Ethics Board.

Study design. Subjects participated in separate EEG and MRI recording sessions on different days (randomized order; 13 subjects experienced the fMRI session first), during which they listened to blocks of repeated speech syllables or piano tones. Before the EEG session, subjects performed a set of computerized behavioral tasks ( $\sim 30 \mathrm{~min})$, including FF discrimination (reported below) and several other measures of musicianship and auditory system function (Nilsson et al., 1994; Foster and Zatorre, 2010) that relate to research questions that are not addressed here.

FF discrimination assessment. FF discrimination thresholds were measured using a 2-interval forced choice task and a 2-down 1-up rule to estimate the threshold at $79 \%$ correct point on the psychometric curve (Levitt, 1971). On each trial, two $250 \mathrm{~ms}$ pure sine tones were presented, separated by $600 \mathrm{~ms}$ of silence. In randomized order, 1 of the 2 tones was a $500 \mathrm{~Hz}$ reference pitch and the other was higher by a percentage that started at 7 and was reduced by 1.25 after 2 correct responses or increased by 1.25 after an incorrect response. The task stopped after 15 reversals and the geometric mean of the last 8 trials was recorded. The task was repeated 5 times and the scores were averaged.

Stimuli. We used two stimuli, a $100 \mathrm{~ms}$ speech syllable (/da/) with a fundamental frequency of $98 \mathrm{~Hz}$ that has been used extensively in previous studies because it elicits clear and replicable responses (Johnson et al., 2005; Skoe and Kraus, 2010; Fig. 1a,b, top) and a piano tone with the same nominal fundamental frequency and stimulus duration, but that had very little energy at the fundamental frequency (McGill University Master Samples database [http://www.worldcat.org/title/mcgill- university-master-samples-collection-on-dvd/oclc/244566561], Steinway piano G2 tone, right channel; Fig. $1 c, d$, top). To ensure that harmonic distortions created by the headphones did not reintroduce energy at the fundamental frequency (Norman-Haignere and McDermott, 2016), we measured sound output from both sets of earphones (S14, Sensimetrics; ER2, Etymotic Research) using a KEMAR Dummy-Head Microphone (www.gras.dk) at the $80 \mathrm{~dB}$ SPL used in the experiment. Although the two earphones yielded slightly different amplitudes for each harmonic component, we found no evidence that energy had been reintroduced at the fundamental frequency.

fMRI data acquisition. The stimulation paradigm took into account the constraints of each of the imaging modalities such that almost identical versions could be presented during the independent EEG and BOLD-fMRI recording sessions. Each interval between scans, defined as a block, comprised a series of 20 stimuli of the same type (interstimulus interval: $\sim 200 \mathrm{~ms}$, jittered by $0-10 \mathrm{~ms}$, randomized), as well as silent breaks (Fig. 2), which were included to reduce the effects of repetition suppression and enhancement that can differ between people (Chandrasekaran et al., 2012). Stimuli were presented binaurally at $80 \pm 1 \mathrm{~dB}$ SPL using a custom-written script (Presentation; Neurobehavioral Systems) using MRI-compatible headphones (S14; Sensimetrics) via foam inserts placed inside the ear canal. Auditory stimulation was timed to maximize the hemodynamic response during fMRI recording to sound 
a
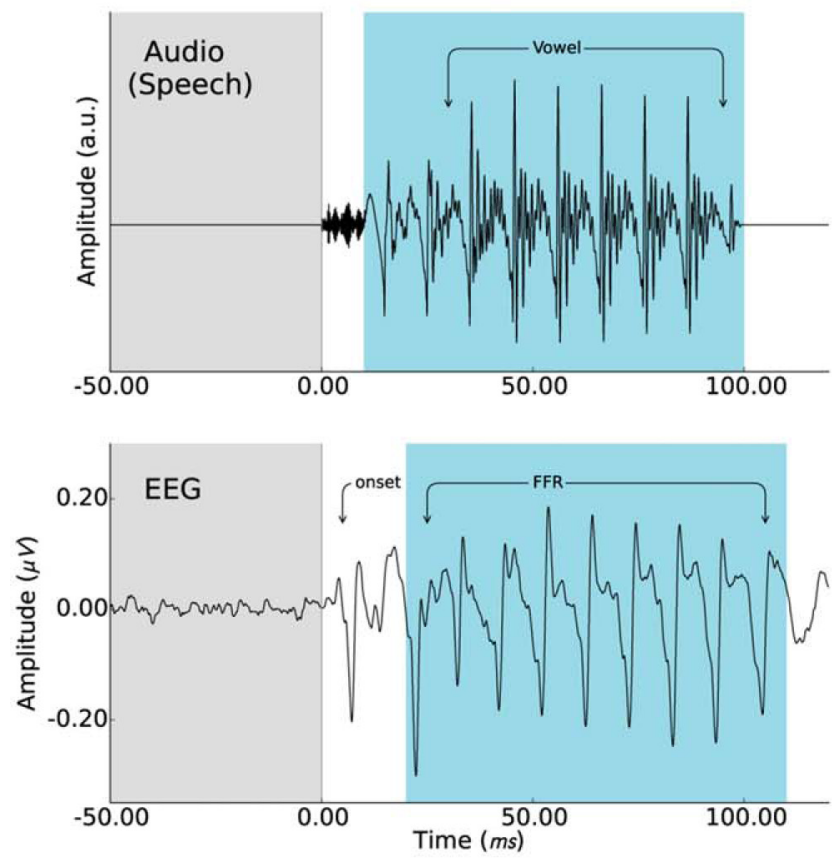

C
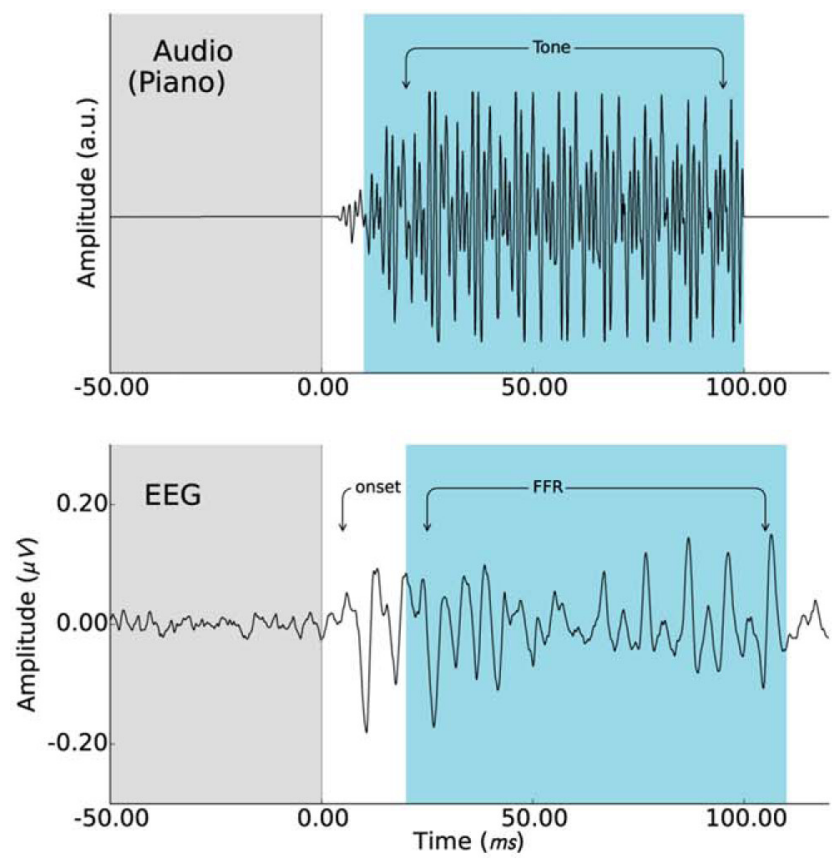

b
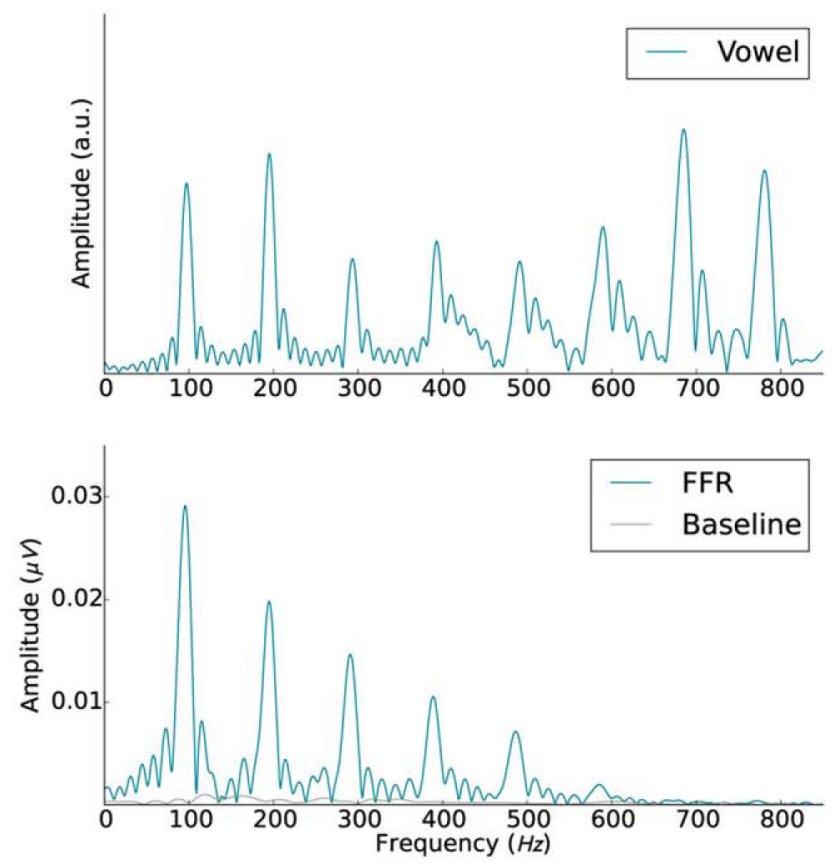

d
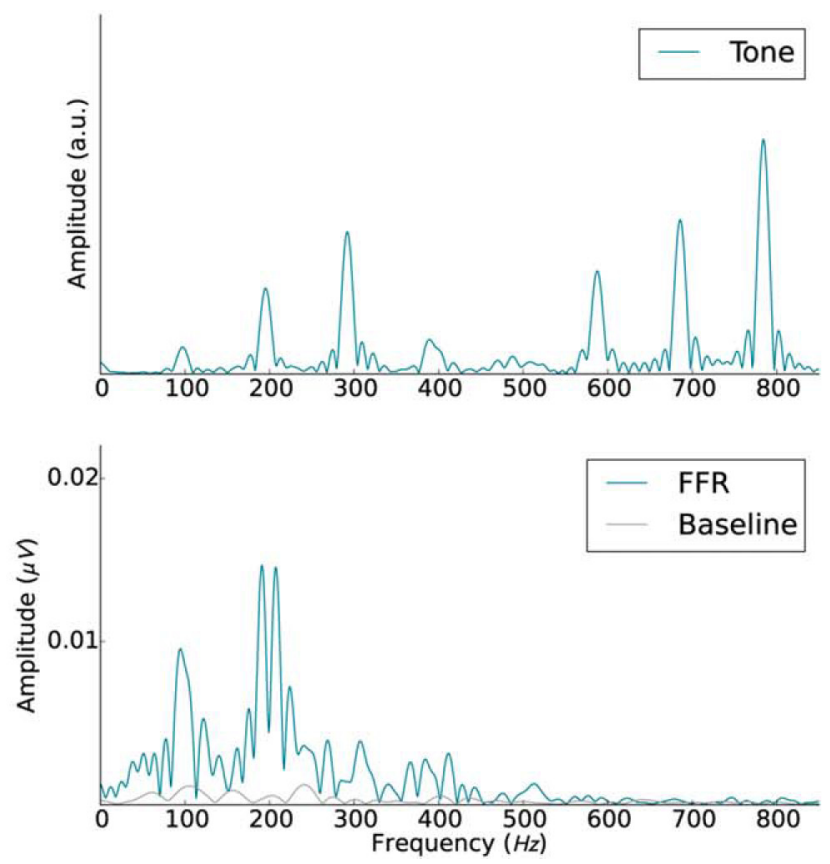

Figure 1. Auditory stimuli and averaged EEG responses. $\boldsymbol{a}, \boldsymbol{b}$, Speech stimulus (syllable: /da/, $98 \mathrm{~Hz}$ fundamental frequency) in the time and frequency domain (top) and the corresponding averaged responses isolated from the EEG recordings (bottom). $\boldsymbol{c}, \boldsymbol{d}$, Tone stimulus (piano "G2," $98 \mathrm{~Hz}$ fundamental frequency) in the time and frequency domain (top) and the EEG responses (bottom). The prestimulus baseline ( -50 to $0 \mathrm{~ms}$ ) and the FFR periods ( $20-110 \mathrm{~ms}$ after sound onset) are marked in gray and blue, respectively.

during the subsequent acquisition (i.e., $\sim 5-7 \mathrm{~s}$ after the onset of the stimulus block), but its exact timing was jittered ( $0-1 \mathrm{~s}$, randomized) to reduce confounds with periodic sources of noise and of top-down expectations. Speech or piano tone blocks were presented pseudorandomly, along with relative silence baseline blocks (for a total of 120 syllable volumes, 120 tone volumes, and 90 baseline volumes). Subjects were asked to listen actively for oddball stimuli ( $80 \%$ normal amplitude) and indicate via button press (right index and middle finger) during the scan after stimulation if one had occurred or not. Oddballs were present in
$30 \%$ of the blocks and replaced 1 of the last 4 stimuli in a block. To control for preparatory motor activity associated with button pressing, baseline volumes included a single stimulus $\sim 1-2 \mathrm{~s}$ from the end of the block to which subjects responded during the scan with a button press. Nine subjects experienced a slight experimental variation in which the single stimulus was presented $\sim 4 \mathrm{~s}$ from the end of the block; this difference was controlled for in each GLM model.

fMRI data were acquired using EPI whole head coverage on a Siemens 3 tesla scanner with a 32-channel head coil at the McConnell Brain Im- 


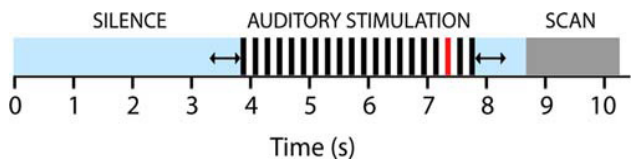

Figure 2. Auditory stimulation paradigm. Each stimulation block consisted of 20 repetitions of the same stimulus (either speech or piano), which was situated within a period of silence and jittered to minimize physiological confounds (see Materials and Methods for details). The same design was used for the EEG and fMRI recording sessions. In $30 \%$ of blocks, a quieter stimulus was presented in place of one of the last four stimuli (indicated in red). Subjects were asked to indicate whether there had been an oddball after each block to control for attention.

aging Center at the Montreal Neurological Institute using a sparse sampling fMRI paradigm (Belin et al., 1999; Hall et al., 1999), which avoids confounding the BOLD signal of interest with effects due to loud noise from gradient switching (voxel size $3.4 \mathrm{~mm}^{3}, 42$ slices, TE $49 \mathrm{~ms}$, TR $\sim 10210 \mathrm{~ms}$ ). We implemented a cardiac gating procedure such that each scan was triggered by the cardiac cycle after the stimulation block (Guimaraes et al., 1998) to address research questions that are not reported here. This resulted in an average block length difference compared with EEG of $\sim 500 \mathrm{~ms}$ and total fMRI scan time was $\sim 1 \mathrm{~h}$ ( 3 runs of 19 min each). To reduce subject fatigue, anatomical MRI scans were acquired between fMRI runs, during which subjects were instructed to lie still and rest.

FMRI analysis. FMRI data were analyzed using FSL software (fMRIB; Smith et al., 2004; Jenkinson et al., 2012). Images were motion corrected, $b_{0}$ unwarped, registered to the T1-weighted anatomical image using boundary-based registration (Greve and Fischl, 2009), and spatially smoothed (5 mm FWHM). Each subjects' anatomical image was registered to MNI $2 \mathrm{~mm}$ standard space (12-parameter linear transformation). For six subjects, gradient field maps had not been acquired; these were substituted by an average of the other 19 subjects' gradient field maps in standard space transformed to native space (12-parameter linear transformation). Task-related BOLD responses of each run were analyzed within GLM (FEAT; Beckmann et al., 2003), including three conditions (relative silence, speech, piano). For each scan, contrast images were computed for speech $>$ relative silence and piano $>$ relative silence and three runs per subject were combined in a fixed-effects model. Within- and between-group analyses were performed using randomeffects models in MNI space (FLAME 1 in FSL; the automatic outlier deweighting option was selected). To test the specific hypotheses of interest and to localize areas of sensitivity to FFR- $f_{0}$ strength within the auditory cortex, a bilateral auditory cortex ROI was defined using the Harvard-Oxford cortical and subcortical structural atlases implemented in FSL: regions with a probability greater or equal to 0.3 of being identified as Heschl's gyrus or planum temporale were included and the resulting ROI was dilated by two voxels to ensure that the central peaks of the cortical signal generators found in previous work (Coffey et al., 2016b) were well within the ROI.

To evaluate the main research questions, we entered the FFR- $f_{0}$ and wave A latency values into a whole-sample GLM model separately for the speech versus relative silence and piano versus relative silence contrast (the minor difference in the silent blocks described above was entered as a covariate of no interest). For multiple-comparisons correction for each research question, we applied voxelwise correction as implemented in FEAT (Gaussian random field-theory-based, $p<0.05$ one-tailed within the bilateral AC ROI; statistical maps thresholded above $Z=2.3$ are presented in Figs. 3 and 4 to show clearly the pattern of results; the position and number of significant voxels are reported in the text). To gain additional evidence that the brain area identified as being sensitive to FFR- $f_{0}$ strength in the speech condition was also related to FFR- $f_{0}$ strength in the piano condition, we ran a conjunction analysis in which the piano condition regression analysis was masked by the significant result from the speech regression analysis. For further analysis of relationships to FF discrimination threshold and musicianship, we extracted a measure of BOLD activity (mean percentage change of parameter estimate) from the small cortical areas that were found to be significantly related to FFR $-f_{0}$ strength in each contrast.
EEG acquisition. Because the EEG version of the paradigm did not include baseline silent blocks nor cardiac gating, the total recording time was $45 \mathrm{~min}$. This was split into three parts, between which short breaks were given. During the recording, subjects sat comfortably in a magnetically shielded room. A Biosemi active electrode system (ActiveTwo) sampled at $16 \mathrm{kHz}$ was used to record EEG from position $\mathrm{Cz}$, with two earlobe references and grounds placed on the forehead above the right eyebrow. Stimuli were presented using a custom-written script (Presentation software; Neurobehavioral Systems) delivered binaurally via insert earphones (ER2; Etymotic Research). Each stimulus was presented 2400 times at alternating polarities to enable canceling of the cochlear microphonic (Skoe and Kraus, 2010). We recorded stimulus onset markers from the stimulus computer along with the EEG data via parallel port. Subjects were asked to keep their bodies and eyes relaxed and still during recordings and were provided with a small picture affixed to the wall as a reminder.

EEG analysis. Data analysis was performed using the EEGLAB toolbox (version 13.5.4b; Delorme and Makeig, 2004), the ERPLAB plugin (version 5.0.0.0), and custom MATLAB scripts (version 7.12.0; The MathWorks, RRID: SCR_001622). Each recording was band-pass filtered (80-2000 Hz; Butterworth fourth order, zero-phase, as implemented in EEGLAB; RRID: SCR_007292), epoched (-50 to $200 \mathrm{~ms}$ around the onset marker), and DC correction was applied to the baseline period. Fifteen percent of epochs having the greatest amplitude were discarded for each subject; this served to remove the majority of epochs contaminated by myogenic activity (confirmed by inspection), yet retain equal numbers of epochs per subject for the computation of phaselocking value (PLV): a measure of FFR strength that is highly correlated with spectral amplitude but that is more statistically sensitive (Zhu et al., 2013). For each subject and stimulus type, a set of 400 epochs from the total pool (2040) was selected randomly with replacement. Each epoch was trimmed to the FFR period (20-110 ms after sound onset), windowed ( $5 \mathrm{~ms}$ raised cosine ramp), zero-padded to $1 \mathrm{~s}$ to allow for a $1 \mathrm{~Hz}$ frequency resolution, and the phase of each epoch was calculated by discrete Fourier transform. The PLV for each epoch was computed by normalizing the complex discrete Fourier transform by its own magnitude and averaging across 1000 iterations. Mean $f_{0}$ strength was taken to be the mean PLV at $f_{0}$ (peak $\pm 2 \mathrm{~Hz}$ ) for each subject and stimulus (see "appendix: analysis methods," item 5, in Zhu et al., 2013 for formulae).

To obtain onset latency, epochs were averaged together by polarity to correct for any effect of the cochlear microphonic (i.e., negative, positive; Wever and Bray, 1930) and summed to form the time domain average. To select an onset peak for analysis, we generated a grand average for each stimulus across all subjects and compared individual waveforms with it, as suggested in Skoe and Kraus (2010); we selected wave A for further analysis for replicability across subjects. An experienced rater who was blind to subject identity and group selected wave A peak latencies for each subject and condition by visual inspection. These were confirmed by a custom automatic algorithm (Spearman's correlation between the manually and automatically selected wave A latency for the speech stimulus: $r_{\mathrm{s}}=0.98, p<0.001$; piano stimulus: $r_{\mathrm{s}}=0.85, p<0.001$ ). Manually selected latencies were deemed to be similar yet were preferred because it was sometimes necessary for the less clear piano onset to select between two local peaks.

Distributions of FFR-derived measures frequently fail tests of normality, as is the case here: we performed Kolmogorov-Smirnov tests on the FFR $-f_{0}$ and wave A latency for each condition and in each case rejected the hypothesis of a normal distribution $(p<0.05)$. Nonparametric statistics were therefore used unless otherwise specified. We compared FFR $-f_{0}$ and wave A latency across musicians and nonmusicians using one-tailed Wilcoxon rank-sum tests and assessed correlations between start age and total practice hours and FFR- $f_{0}$ strength and wave A latency using Spearman's $\rho, r_{\mathrm{s}}$.

Anatomical data. Between the first and second functional imaging run, we recorded whole-head anatomical T1-weighted images (MPRAGE, voxel size $1 \mathrm{~mm}^{3}$ ). FreeSurfer was used to automatically segment each brain (Fischl et al., 2002; RRID: SCR_001847). Between the second and third run, we recorded diffusion-weighted images (DWIs; 99 directions, voxel size $2.0 \mathrm{~mm}^{3}$, 72 slices, TE $88 \mathrm{~ms}$, TR $9340 \mathrm{~ms}, b=1000 \mathrm{~s} / \mathrm{mm}^{2}$ ). 
a

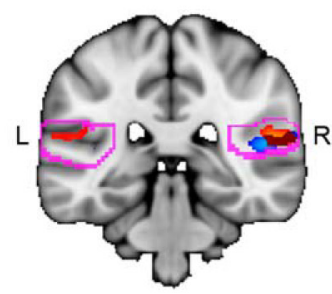

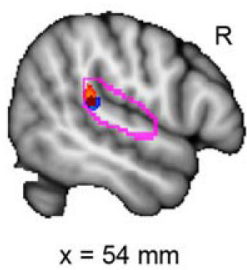

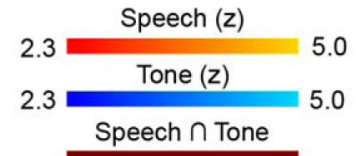

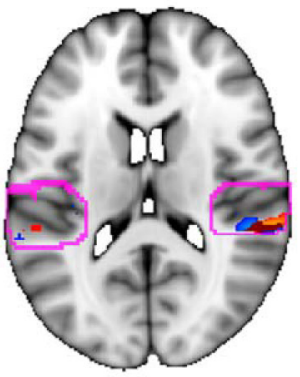

$z=15 \mathrm{~mm}$ b

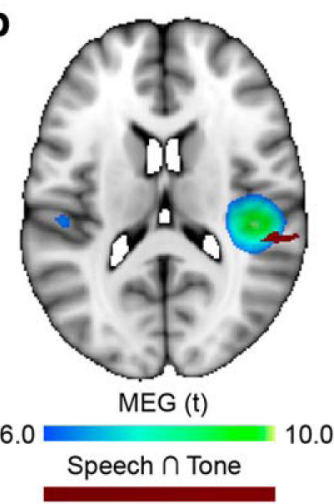

Figure 3. Areas within the auditory cortex that are sensitive to FFR $-f_{0}$ strength. $\boldsymbol{a}$, Coronal, sagittal, and horizontal brain slices showing statistical maps where BOLD signal was related to FFR- $f_{0}$ strength for each stimulus set; greater FFR strength was related to higher BOLD signal in the right planum temporale (speech: orange; piano: blue). Overlapping regions are indicated in maroon. Bilateral ROIs encompassing the auditory cortex bilaterally are delineated in pink. $\boldsymbol{b}$, Horizontal slice showing the location of $\mathrm{FFR}-f_{0}$ sensitive cortex in both conditions (maroon) in relation to the previous result of a right auditory cortex contribution to the FFR- $f_{0}$ from MEG (Coffey et al., 2016b). Note that fMRI and MEG differ in their spatial resolution.

DWIs were corrected for eddy current distortions, brains were extracted from unweighted images, and a diffusion tensor model was fit using FSL's "dtifit" function to obtain voxelwise maps of the diffusion parameters [FA, mean diffusivity (MD), and axial diffusivity (AD); RRID: SCR_002823]. Radial diffusivity (RD) was calculated as the mean of the second and third eigenvalues of the diffusion tensor.

ROIs below the gray matter that were identified as Heschl's gyrus and sulcus by Freesurfer segementation (Destrieux et al., 2010) were created for each hemisphere by transforming surface labels from each participant's native space into their diffusion-weighted volume space, projecting the labels to a depth of $2 \mathrm{~mm}$ (parallel to the cortical surface) and visually confirming that voxels lay in white matter for each participant; these masks are used to address questions of lateralization and relationships to fMRI and EEG results in white matter that is most directly related to the auditory cortex (Shiell and Zatorre, 2016). Transformation matrices were calculated between DWI space and structural space (T1-weighted image, FLIRT, 6 degrees of freedom) and to a $1 \mathrm{~mm}$ FA template (FMRIB58_FA_1 mm, FLIRT, 12 degrees of freedom), concatenated, and their inverses used to transform individual Heschl's gyrus and sulcus masks to diffusion space to extract diffusion measures.

To address research questions about possible differences in the microstructure of white matter underlying regions of the auditory cortex that were found to be sensitive to onset timing, we first evaluated correlations between onset latency in the speech condition and two measures of white matter microstructure, FA, and MD, in each white matter ROI (corrected for multiple comparisons, $\alpha=0.05 / 4$ ). To better understand the MD result, we also assessed correlations between onset latency in the speech condition and subcomponents of $\mathrm{MD}: \mathrm{AD}$ and $\mathrm{RD}$. To assess the lateralization of the observed MD finding, we compared the correlations in each auditory cortex statistically using Fisher's $r$-to- $Z$ transformation (Steiger, 1980). Finally, we predicted a negative correlation between BOLD response and MD values in the left auditory cortex based on the BOLD-onset and onset-MD correlations and tested this relationship for statistical significance using Spearman's $\rho$ (one-tailed).

\section{Results}

\section{Attention control}

Subjects correctly identified most of the blocks as either containing oddball (quieter) stimuli or not during both sessions (EEG mean accuracy $=85.3 \%, \mathrm{SD}=11.2$; fMRI mean accuracy $=$
$90.9 \%$, SD $=8.7$ ); this served to confirm that subjects were attending to the stimuli.

\section{Regression of FFR- $f_{0}$ with BOLD-fMRI data}

Speech condition

In the speech $>$ relative silence contrast, FFR- $f_{0}$ strength was significantly correlated with BOLD signal in the right (but not left) posterior auditory cortex/planum temporale (Fig. $3 a, b$; the group of significant voxels has a volume of $128 \mathrm{~mm}^{3}$ and is centered at: $x=60, y=-34, z=14 \mathrm{~mm} ; 2 \mathrm{~mm}$ MNI152 space; $Z=$ 3.99). Musicians showed significantly stronger BOLD responses than nonmusicians within the region identified as being significantly sensitive to FFR- $f_{0}$ strength [Wilcoxon rank-sum test, one-tailed: $Z=2.15, p=0.016$; musician mean: $0.53 \%$ change of parameter estimate $(\mathrm{SD}=0.50)$; nonmusician mean: $0.16 \%$ $(\mathrm{SD}=0.47)$, although the between-group differences in FFR- $f_{0}$ strength did not reach significance $(Z=0.24, p=0.4$; musician mean PLV: 0.14; SD $=0.06)$; nonmusician mean PLV: 0.12 $(\mathrm{SD}=0.04)]$.

\section{Piano condition}

In the piano $>$ relative silence contrast, $\mathrm{FFR}-f_{0}$ was significantly correlated with BOLD signal in the right $\mathrm{AC}$ region (the group of significant voxels has a volume of $112 \mathrm{~mm}^{3}$ and is centered at: $x=$ 52, $y=-34, z=12 \mathrm{~mm} ; 2 \mathrm{~mm}$ MNI152 space; $Z=4.10$; Fig. 3 ). 
a

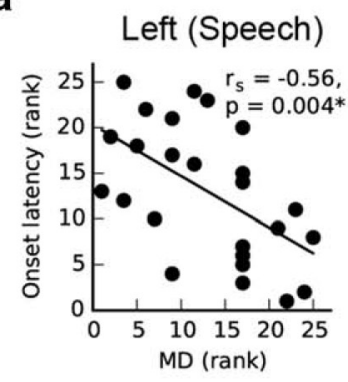

b

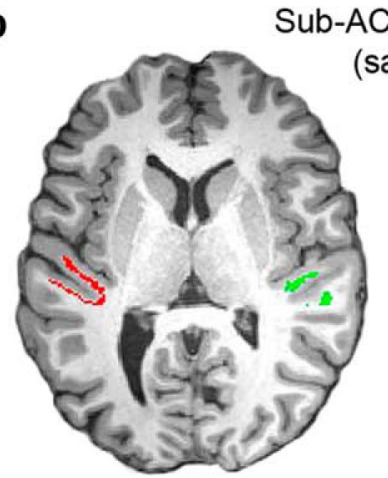

C (sample subject)

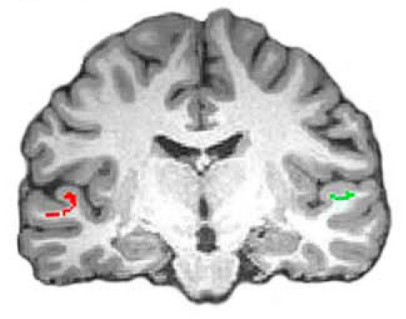

Right (Speech)

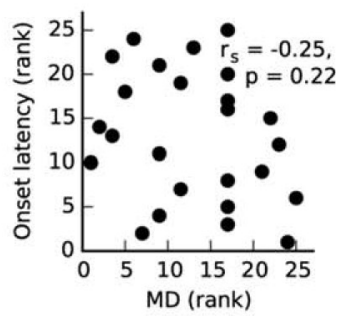

Figure 5. White matter microstructure is related to onset latency. $\boldsymbol{a}$, Left hemisphere MD values within anatomically defined ROIs show a significant correlation: shorter latencies were related to greater MD values. $\boldsymbol{b}$, White matter ROIs underlying auditory cortex on a single example subject overlaid on a T1-weighted anatomical image for illustrative purposes. $\boldsymbol{c}$, Similar analyses on the right side did not show any significant correlation. This analysis was performed only in the speech condition because onset latencies to the natural piano tone were more variable (see Materials and Methods and Results for details).

The conjunction analysis revealed that the majority of the region identified as sensitive to FFR- $f_{0}$ in the Speech condition was also significantly related to FFR- $f_{0}$ in the Piano condition (i.e., 112 $\mathrm{mm}^{3}$ of $128 \mathrm{~mm}^{3}$ ). As in the speech condition, musicians showed significantly stronger BOLD responses than nonmusicians within the region identified as being significantly sensitive to FFR- $f_{0}$ strength [Wilcoxon rank-sum test: $Z=2.15, p=0.016$; musician mean: $0.37 \%(\mathrm{SD}=0.54)$; nonmusician mean: $0.07 \%$ $(\mathrm{SD}=0.44)]$. The between-group differences in FFR- $f_{0}$ strength did not reach significance, although a trend was suggested $[Z=$ 1.50, $p=0.067$; musician mean PLV: 0.09 (SD = 0.04); nonmusician mean PLV: $0.07(\mathrm{SD}=0.02)$ ].

In addition to the right $\mathrm{AC}$ area, several voxels within the left hemisphere ROI at the extreme anterior end were found to be significantly related to FFR- $f_{0}$ strength. This region does not overlap with the left auditory cortex FFR- $f_{0}$ generator derived from the MEG, nor does it appear to be in homologous regions the right auditory cortex finding, but for completeness, we explored this finding by inspecting the statistical maps from each condition in the vicinity of the ROI borders. The left anterior group of significant voxels was located in the posterior division of the superior temporal sulcus (center: $x=-66, y-18, z=-2$ $\mathrm{mm} ; 2 \mathrm{~mm}$ MNI152 standard brain; $Z=4.1$ ). A similar group of significant voxels was also present in the speech versus relative silence condition (maximum: $x=-58, y=-16, z=-4 \mathrm{~mm}$, $Z=2.73$ ). One additional group was found in the left posterior parietal operculum outside of the ROI (piano $>$ relative silence condition: $x=-46, y=-40, z=24 \mathrm{~mm} ; Z=2.78$; speech $>$ relative silence condition: $x=-48, y=-40, z=26 \mathrm{~mm} ; Z=$ $3.52)$. Significant voxels did not appear in the right hemisphere homolog structures in either condition, nor did there appear to be other $f_{0}$-sensitive areas near the right hemisphere ROI borders.

\section{Regression of onset latency with BOLD-fMRI data \\ Speech condition}

In the speech $>$ relative silence contrast, longer-wave A latencies were correlated with greater BOLD signal in the left (but not right) Heschl's sulcus (Fig. 4; the group of significant voxels has a volume of $40 \mathrm{~mm}^{3}$ and is centered at: $x=-42, y=-32, z=6$ $\mathrm{mm} ; 2 \mathrm{~mm}$ MNI152 space; $Z=4.29$; Fig. $5 a, c)$. BOLD signal was not significantly related to shorter latencies, which are considered to index better functioning, in any regions. We did not observe a difference between musicians and nonmusicians in BOLD response within the area sensitive to wave A latency (Wilcoxon rank-sum test: $Z=-0.73, p=0.46)$, nor in the wave A latency values $(Z=-0.41, p=0.34)$.

\section{Piano condition}

No areas were significantly related to piano wave A onset latency in the piano $>$ relative silence contrast. Although a subthreshold peak was observed within the area sensitive to latency in the speech condition $(x=-42, y=-32, z=10 ; Z=2.35 ; 2 \mathrm{~mm}$ MNI152; see the conjunction (green) in Fig. 4 for location), we performed secondary analyses relating to onset latency only in the significant speech condition.

\section{Onset latency and microstructure of white matter underlying auditory cortex}

Onset latency in the speech condition was significantly correlated with average $\mathrm{MD}$ values within the white matter ROI underlying Heschl's gyrus and sulcus in the left hemisphere (two-tailed, corrected for multiple comparisons, $\alpha=0.05 / 4 ; r_{\mathrm{s}}=-0.56, p=$ 0.004; Fig. $5 a)$, but not in the right hemisphere $\left(r_{\mathrm{s}}=-0.25, p=\right.$ 0.22 ; Fig. $5 c$ ). The correlation between onset latency and MD was significantly greater in the left than the right hemisphere (Fisher's $r$-to- $z$ transformation, one-tailed, $Z=1.765, p=0.039$ ). Significant relationships between onset latency and mean FA were not observed in the left hemisphere ROI $\left(r_{\mathrm{s}}=-0.19, p=0.36\right)$ nor the right hemisphere ROI $\left(r_{\mathrm{s}}=-0.10, p=0.62\right)$.

Both $\mathrm{AD}$ and $\mathrm{RD}$ showed similar patterns in their relationships to onset latency, as did MD in the left hemisphere (AD vs onset: $r_{\mathrm{s}}=-0.62, p=0.0008$; RD vs onset latency: $r_{\mathrm{s}}=-0.45$, $p=0.024)$ and no significant relationship in both cases in the right hemisphere (AD vs onset: $r_{\mathrm{s}}=-0.24, p=0.25$; RD vs onset latency: $\left.r_{\mathrm{s}}=-0.22, p=0.29\right)$.

If a greater BOLD response and lower MD are both indices of neural conduction inefficiency, then we would predict a negative correlation between MD under left Heschl's gyrus and BOLD response in the overlying gray matter. This is in fact the case $\left(r_{\mathrm{s}}=\right.$ $-0.42, p=0.019)$. Musicians did not differ significantly from nonmusicians in $\mathrm{MD}$ on either side (reported values are twotailed; left: $Z=0, p=1.0$; right: $Z=0.14, p=0.89$ ).

\section{FF discrimination}

Assessment of FF discrimination skills

The mean FF discrimination threshold was $1.40 \%$ overall (SD = 1.45). Musicians had lower FF discrimination thresholds than nonmusicians, as expected (musician mean: $0.58 \%, \mathrm{SD}=0.37$; 
nonmusician mean: $2.29 \%, \mathrm{SD}=1.66 ; Z=3.40, p<0.001$ ). The BOLD signal strength extracted from the FFR- $f_{0}$-sensitive region was not significantly correlated with FF discrimination (reported $p$-values are one-tailed; speech condition: $r_{\mathrm{s}}=-0.18, p=0.19$; piano condition: $\left.r_{\mathrm{s}}=-0.13, p=0.27\right)$, nor was frequency discrimination and BOLD signal significantly related within the FFR $f_{0}$-sensitive auditory cortex regions (speech condition: $r_{\mathrm{s}}=$ $-0.31, p=0.06$; piano condition: $r_{\mathrm{s}}=-0.24, p=0.12$ ).

\section{Discussion}

Our results demonstrate that hemodynamic activity in the right posterior auditory cortex is sensitive to FFR- $f_{0}$ strength, a finding that was replicated in two separate stimulus sets with and without energy at the fundamental frequency and conforms to predictions arising from our prior MEG study (Coffey et al., 2016b). The right-lateralized FFR- $f_{0}$-sensitive region was dissociable from a left-lateralized region in Heschl's sulcus that was sensitive to the latency of the onset response. This finding was further supported by a significant relationship between onset latency and the microstructure of the white matter immediately underlying primary auditory areas in the left (but not right) hemisphere and a significant correlation between BOLD response in the onset-sensitive region and $\mathrm{MD}$ in underlying white matter. A lateralization of the relationship between onset timing and white matter microstructure is supported by a direct comparison of correlation strength.

\section{Relationship between BOLD-fMRI and FFR- $f_{0}$}

Our primary aim was to adduce evidence in favor of a cortical source for the FFR (Musacchia et al., 2008; Coffey et al., 2016b), to which end we tested the hypothesis that the FFR- $f_{0}$ strength is correlated with fMRI signal in the right auditory cortex. We reasoned that, if interindividual variations in $\mathrm{FFR}-f_{0}$ strength reflect differences in the coherence or number of phase-locked neurons within this population, then these variations should be paralleled by differences in localized metabolic requirements that would manifest as an FFR $-f_{0}$-sensitive area in the fMRI signal. This hypothesis was supported by and further corroborates preliminary reports of an FFR-like signal measured intracranially from the auditory cortex (Bellier et al., 2014). Together with previous MEG work (Coffey et al., 2016b), our data suggest that findings based on the FFR $-f_{0}$ should not be assumed to have purely brainstem origins. Because these findings are in agreement with the conclusion based on MEG data that there is a cortical component to the FFR, they also support the use of the new MEG-FFR method to observe the sources of the more commonly used scalprecorded EEG-FFR.

That two independent stimuli result in overlapping areas of FFR- $f_{0}$ sensitivity, regardless of whether $f_{0}$ energy is present in the auditory signal, suggests that the sound representation within this region may be involved in computation of pitch at an abstract level. Missing fundamental stimuli are known to produce FFRs with energy at the fundamental frequency (Smith et al., 1978; Galbraith, 1994) and interindividual variability in $f_{0}$ strength is related to interindividual variability and conscious control of missing fundamental perception, although not in a linear manner (Coffey et al., 2016a). Together, these results raise the possibility that top-down task modulation and perhaps experience-related modulation of FFR $-f_{0}$ strength observed previously (Musacchia et al., 2007; Lehmann and Schönwiesner, 2014) could occur at the level of the auditory cortex, although it does not rule out the possibility that the strength of subcortical FFR $-f_{0}$ components are also modulated concurrently. The right auditory cortex has been implicated previously in missing fundamental pitch computation (Schneider and Wengenroth, 2009): patients with right temporal-lobe excisions that include the right lateral auditory cortex have difficulty perceiving the missing fundamental (Zatorre, 1988) and asymmetry in gray matter volume in lateral Heschl's gyrus is related to pitch perception bias (Patel and Balaban, 2001; Schneider et al., 2005). Although the FFR- $f_{0}$ is likely not a direct representation of pitch (Gockel et al., 2011), our results further connect the FFR's pitch-bearing information to processes taking place in auditory cortex regions that represent pitch in an invariant fashion (Penagos et al., 2004; Bendor and Wang, 2006; Norman-Haignere et al., 2013).

\section{Relationship between BOLD-fMRI and onset response latency}

The onset response and the FFR- $f_{0}$ may be represented in different auditory streams (Kraus and Nicol, 2005) because each measure covaries with distinct behavioral and clinical measures (Kraus and Nicol, 2005; Skoe and Kraus, 2010); we therefore wanted to test for a dissociation in the cortical areas sensitive to each measure. However, the mechanistic basis for predicting a greater fMRI signal with a greater amplitude (as in the FFR- $f_{0}$ analysis) does not hold true for latencies; we do not expect shorter onset latencies to necessarily relate to a larger population of neurons firing and therefore greater metabolic requirements that would be reflected in the BOLD signal, nor could onset-related sensitivity be directly related to the generation of the onset response, which occurs in the brainstem before sufficient time has elapsed for neural transmission to the cortex (Parkkonen et al., 2009). We therefore tested both positive and negative relationships. We found only a significant negative relationship: greater BOLD responses are related to longer latencies in left auditory cortex.

To confirm this result and partly inform a mechanistic explanation, we investigated the microstructure of white matter in ROIs directly underlying Heschl's gyrus and sulcus. In a study of the relations between task-related BOLD signal in human gray matter and measures of white matter microstructure, Burzynska et al. (2013) reported that greater microstructural integrity of major white matter tracts was negatively related to BOLD signal, which was interpreted as better quality of structural connections allowing for more efficient use of cortical resources. If a similar mechanism is at work here, then we would expect that the BOLD sensitivity to onset latency should be paralleled by a relationship between WM microstructure and onset latency and this relationship should also show a left lateralization. We confirmed these relationships in the MD measure (corroborated in radial and AD subcomponents), but not the FA measure. FA is a measure of relative degree of sphericity versus linearity of the diffusion tensor, which may not be as relevant a measure in white matter underlying GM as in major white matter tracts due to the presence of association fibers. Although the nature of the observed structural sensitivity to onset latency in the white matter at the cellular level cannot be ascertained from diffusion-weighted data, the direction of the observed relationships among onset latency, BOLD signal, and diffusivity suggests that lower MD in white matter and lower BOLD response in overlying areas are associated with greater neural conduction efficiency within the ascending white matter pathways that carry the onset signal to the cortex. Further work is needed to confirm the white matter finding reported here and to clarify whether it reflects more extensive white matter differences throughout 
the ascending auditory pathway, as would be predicted by the relationship to the timing of the subcortically generated onset response.

\section{Relative lateralization}

We found a right-lateralized relationship between BOLD signal and FFR- $f_{0}$ and a left-lateralized relationship between BOLD signal and onset latency (which was supported by a lateralization in underlying white matter structure). Our results are in agreement with previous evidence of a relative specialization of the right AC for aspects of pitch and tonal processing (Zatorre, 1988; Zatorre and Belin, 2001; Patterson et al., 2002; Hyde et al., 2008; Mathys et al., 2010; Albouy et al., 2013; Herholz et al., 2016; Matsushita et al., 2015; Cha et al., 2016). There is also experimental evidence for a complementary left AC specialization for aspects of temporal resolution (for review, see Zatorre et al., 2002; Poeppel, 2003; Wong et al., 2008), although the interpretation of such findings and how they relate to linguistic processes is controversial (Scott and McGettigan, 2013). Nonetheless, the pattern of results reported here, particularly that onset response timing is related to both BOLD response in primary auditory cortex gray matter and in the structural properties of underlying white matter in the left but not right hemisphere, does favor the proposal of a relative specialization for enhanced temporal resolution in the left auditory cortex. Further work is needed to determine where in the lower levels of the auditory system this lateralization first emerges.

\section{Relationship to training and behavior}

We found that the BOLD signal was significantly greater in musicians for both stimuli within the FFR- $f_{0}$-sensitive area, which is consistent with several prior studies (Pantev and Herholz, 2011) and likely reflects enhanced processing of pitch information. We found significant effects of musician training in the fMRI data. Although the FFR $-f_{0}$ effects do not reach significance, differences have not been observed consistently in similar sample sizes (Musacchia et al., 2007; Wong et al., 2007; Lee et al., 2009; Strait et al., 2012), possibly because they may be eclipsed by large interindividual variations (Coffey et al., 2016a). Previous work also showed clearer behavioral relationships to FFR- $f_{0}$ components that had been separated by their source using MEG than to the FFR- $f_{0}$ strength measured with EEG (Coffey et al., 2016b); it is therefore possible that the compound nature of the EEG signal obscures behavioral relationships of interest here.

\section{Conclusion}

Our results validate and extend the prediction from MEG data of a right auditory cortex contribution to the FFR and show a dissociation in early cortical auditory regions of the FFR $-f_{0}$ and onset timing, providing further evidence that the auditory cortex is both functionally and structurally lateralized. The finding that interindividual differences in FFR strength and onset latency in a population of normal-hearing young adults have cortical correlates supports the idea that these measures represent variations in input quality to different higher-level cortical functions and processing streams, which in turn influences perception and behavior.

\section{References}

Albouy P, Mattout J, Bouet R, Maby E, Sanchez G, Aguera PE, Daligault S, Delpuech C, Bertrand O, Caclin A, Tillmann B (2013) Impaired pitch perception and memory in congenital amusia: the deficit starts in the auditory cortex. Brain 136:1639-1661. CrossRef Medline

Attal Y, Schwartz D (2013) Assessment of subcortical source localization using deep brain activity imaging model with minimum norm operators: a MEG study. PLoS One 8:e59856. CrossRef Medline

Beckmann CF, Jenkinson M, Smith SM (2003) General multilevel linear modeling for group analysis in FMRI. Neuroimage 20:1052-1063. CrossRef Medline

Belin P, Zatorre RJ, Hoge R, Evans AC, Pike B (1999) Event-related fMRI of the auditory cortex. Neuroimage 10:417-429. CrossRef Medline

Bellier L, Bidet-Caulet A, Bertrand O, Thai-Van H, Caclin A (2014) Auditory brainstem responses in the human auditory cortex?! Evidence from sEEG. Poster presented at 20th Annual Meeting of the Organization for Human Brain Mapping. Hamburg, Germany.

Bendor D, Wang X (2006) Cortical representations of pitch in monkeys and humans. Curr Opin Neurobiol 16:391-399. CrossRef Medline

Bidelman GM (2013) The role of the auditory brainstem in processing musically relevant pitch. Front Psychol 4:264. CrossRef Medline

Bones O, Hopkins K, Krishnan A, Plack CJ (2014) Phase locked neural activity in the human brainstem predicts preference for musical consonance. Neuropsychologia 58:23-32. CrossRef Medline

Burzynska AZ, Garrett DD, Preuschhof C, Nagel IE, Li SC, Bäckman L, Heekeren HR, Lindenberger U (2013) A scaffold for efficiency in the human brain. J Neurosci 33:17150-17159. CrossRef Medline

Cha K, Zatorre RJ, Schönwiesner M (2016) Frequency selectivity of voxelby-voxel functional connectivity in human auditory cortex. Cereb Cortex 26:211-224. CrossRef Medline

Chandrasekaran B, Kraus N (2010) The scalp-recorded brainstem response to speech: neural origins and plasticity. Psychophysiology 47:236-246. CrossRef Medline

Chandrasekaran B, Kraus N, Wong PC (2012) Human inferior colliculus activity relates to individual differences in spoken language learning. J Neurophysiol 107:1325-1336. CrossRef Medline

Coffey EBJ, Herholz SC, Scala S, Zatorre RJ (2011) Montreal Music History Questionnaire: a tool for the assessment of music-related experience in music cognition research. Poster presented at The Neurosciences and Music IV: Learning and Memory Conference. Edinburgh, UK.

Coffey EBJ, Colagrosso EM, Lehmann A, Schönwiesner M, Zatorre RJ (2016a) Individual differences in the frequency-following response: Relation to pitch perception. PLoS One 11:e0152374. CrossRef Medline

Coffey EBJ, Herholz SC, Chepesiuk AM, Baillet S, Zatorre RJ (2016b) Cortical contributions to the auditory frequency-following response revealed by MEG. Nat Commun 7:11070. CrossRef Medline

Delorme A, Makeig S (2004) EEGLAB: an open source toolbox for analysis of single-trial EEG dynamics including independent component analysis. J Neurosci Methods 134:9-21. CrossRef Medline

Destrieux C, Fischl B, Dale A, Halgren E (2010) Automatic parcellation of human cortical gyri and sulci using standard anatomical nomenclature. Neuroimage 53:1-15. CrossRef Medline

Fischl B, Salat DH, Busa E, Albert M, Dieterich M, Haselgrove C, van der Kouwe A, Killiany R, Kennedy D, Klaveness S, Montillo A, Makris N, Rosen B, Dale AM (2002) Whole brain segmentation. Neuron 33:341355. CrossRef Medline

Foster NE, Zatorre RJ (2010) Cortical structure predicts success in performing musical transformation judgments. Neuroimage 53:26-36. CrossRef Medline

Galbraith GC (1994) Two-channel brain-stem frequency-following responses to pure tone and missing fundamental stimuli. Electroencephalogr Clin Neurophysiol 92:321-330. CrossRef Medline

Galbraith GC, Arroyo C (1993) Selective attention and brainstem frequency-following responses. Biol Psychol 37:3-22. CrossRef Medline

Gockel HE, Carlyon RP, Mehta A, Plack CJ (2011) The frequency following response (FFR) may reflect pitch-bearing information but is not a direct representation of pitch. J Assoc Res Otolaryngol 12:767-782. CrossRef Medline

Greve DN, Fischl B (2009) Accurate and robust brain image alignment using boundary-based registration. Neuroimage 48:63-72. CrossRef Medline

Guimaraes AR, Melcher JR, Talavage TM, Baker JR, Ledden P, Rosen BR, Kiang NY, Fullerton BC, Weisskoff RM (1998) Imaging subcortical auditory activity in humans. Hum Brain Mapp 6:33-41. CrossRef Medline

Hall DA, Haggard MP, Akeroyd MA, Palmer AR, Summerfield AQ, Elliott MR, Gurney EM, Bowtell RW (1999) Sparse temporal sampling in auditory fMRI. Hum Brain Mapp 7:213-223. CrossRef Medline

Herholz SC, Coffey EBJ, Pantev C, Zatorre RJ (2016) Dissociation of neural 
networks for predisposition and for training-related plasticity in auditory-motor learning. Cereb Cortex 26:3125-3134. CrossRef Medline

Hoormann J, Falkenstein M, Hohnsbein J, Blanke L (1992) The human frequency-following response (FFR): normal variability and relation to the click-evoked brainstem response. Hear Res 59:179-188. CrossRef Medline

Hyde KL, Peretz I, Zatorre RJ (2008) Evidence for the role of the right auditory cortex in fine pitch resolution. Neuropsychologia 46:632-639. CrossRef Medline

Jenkinson M, Beckmann CF, Behrens TE, Woolrich MW, Smith SM (2012) FSL. Neuroimage 62:782-790. CrossRef Medline

Johnson KL, Nicol TG, Kraus N (2005) Brain stem response to speech: a biological marker of auditory processing. Ear Hear 26:424-434. CrossRef Medline

Kraus N, Nicol T (2005) Brainstem origins for cortical "what" and "where" pathways in the auditory system. Trends Neurosci 28:176-181. CrossRef Medline

Kraus N, White-Schwoch T (2015) Unraveling the biology of auditory learning: a cognitive-sensorimotor-reward framework. Trends Cogn Sci 19:642-654. CrossRef Medline

Krishnan A (2007) Human frequency following response. In: Auditory evoked potentials: basic principles and clinical application (Burkard RF, Don M, Eggermont JJ, eds), pp 313-335. Baltimore: Lippincott Williams and Wilkins.

Krishnan A, Swaminathan J, Gandour JT (2009) Experience-dependent enhancement of linguistic pitch representation in the brainstem is not specific to a speech context. J Cogn Neurosci 21:1092-1105. CrossRef Medline

Lee KM, Skoe E, Kraus N, Ashley R (2009) Selective subcortical enhancement of musical intervals in musicians. J Neurosci 29:5832-5840. CrossRef Medline

Lehmann A, Schönwiesner M (2014) Selective attention modulates human auditory brainstem responses: relative contributions of frequency and spatial cues. PLoS One 9:e85442. CrossRef Medline

Levitt H (1971) Transformed up-down methods in psychoacoustics. J Acoust Soc Am 49:467-477. CrossRef

Magri C, Schridde U, Murayama Y, Panzeri S, Logothetis NK (2012) The amplitude and timing of the BOLD signal reflects the relationship between local field potential power at different frequencies. J Neurosci 32: 1395-1407. CrossRef Medline

Mathys C, Loui P, Zheng X, Schlaug G (2010) Non-invasive brain stimulation applied to Heschl's gyrus modulates pitch discrimination. Front Psychol 1:193. CrossRef Medline

Matsushita R, Andoh J, Zatorre RJ (2015) Polarity-specific transcranial direct current stimulation disrupts auditory pitch learning. Front Neurosci 9:174. CrossRef Medline

Musacchia G, Sams M, Skoe E, Kraus N (2007) Musicians have enhanced subcortical auditory and audiovisual processing of speech and music. Proc Natl Acad Sci U S A 104:15894-15898. CrossRef Medline

Musacchia G, Strait D, Kraus N (2008) Relationships between behavior, brainstem and cortical encoding of seen and heard speech in musicians and non-musicians. Hear Res 241:34-42. CrossRef Medline

Nilsson M, Soli SD, Sullivan J (1994) Development of the hearing in noise test for the measurement of speech reception thresholds in quiet and in noise. J Acoust Soc Am 95:1085-1099. CrossRef Medline

Norman-Haignere S, McDermott JH (2016) Distortion products in auditory fMRI research: measurements and solutions. Neuroimage 129:401413. CrossRef Medline

Norman-Haignere S, Kanwisher N, McDermott JH (2013) Cortical pitch regions in humans respond primarily to resolved harmonics and are located in specific tonotopic regions of anterior auditory cortex. J Neurosci 33:19451-19469. CrossRef Medline

Pantev C, Herholz SC (2011) Plasticity of the human auditory cortex related to musical training. Neurosci Biobehav Rev 35:2140-2154. CrossRef Medline

Parkkonen L, Fujiki N, Mäkelä JP (2009) Sources of auditory brainstem responses revisited: contribution by magnetoencephalography. Hum Brain Mapp 30:1772-1782. CrossRef Medline

Patel AD, Balaban E (2001) Human pitch perception is reflected in the tim- ing of stimulus-related cortical activity. Nat Neurosci 4:839-844. CrossRef Medline

Patterson RD, Uppenkamp S, Johnsrude IS, Griffiths TD (2002) The processing of temporal pitch and melody information in auditory cortex. Neuron 36:767-776. CrossRef Medline

Penagos H, Melcher JR, Oxenham AJ (2004) A neural representation of pitch salience in nonprimary human auditory cortex revealed with functional magnetic resonance imaging. J Neurosci 24:6810-6815. CrossRef Medline

Poeppel D (2003) The analysis of speech in different temporal integration windows: cerebral lateralization as "asymmetric sampling in time." Speech Communication 41:245-255.

Ruggles D, Bharadwaj H, Shinn-Cunningham BG (2012) Why middle-aged listeners have trouble hearing in everyday settings. Curr Biol 22:14171422. CrossRef Medline

Schneider P, Wengenroth M (2009) The neural basis of individual holistic and spectral sound perception. Contemporary Music Review 28:315-328. CrossRef

Schneider P, Sluming V, Roberts N, Scherg M, Goebel R, Specht HJ, Dosch HG, Bleeck S, Stippich C, Rupp A (2005) Structural and functional asymmetry of lateral Heschl's gyrus reflects pitch perception preference. Nat Neurosci 8:1241-1247. CrossRef Medline

Scott SK, McGettigan C (2013) Do temporal processes underlie left hemisphere dominance in speech perception? Brain Lang 127:36-45. CrossRef Medline

Shiell MM, Zatorre RJ (2016) White matter structure in the right planum temporale region correlates with visual motion detection thresholds in deaf people. Hear Res. In press.

Skoe E, Kraus N (2010) Auditory brain stem response to complex sounds: a tutorial. Ear Hear 31:302-324. CrossRef Medline

Smith JC, Marsh JT, Greenberg S, Brown WW (1978) Human auditory frequency-following responses to a missing fundamental. Science 201: 639-641. CrossRef Medline

Smith SM, Jenkinson M, Woolrich MW, Beckmann CF, Behrens TE, Johansen-Berg H, Bannister PR, De Luca M, Drobnjak I, Flitney DE, Niazy RK, Saunders J, Vickers J, Zhang Y, De Stefano N, Brady JM, Matthews PM (2004) Advances in functional and structural MR image analysis and implementation as FSL. Neuroimage 23:S208-S219. CrossRef Medline

Steiger JH (1980) Tests for comparing elements of a correlation matrix. Psychological Bulletin 87:245-251. CrossRef

Strait DL, Kraus N, Skoe E, Ashley R (2009) Musical experience promotes subcortical efficiency in processing emotional vocal sounds. Ann N Y Acad Sci 1169:209-213. CrossRef Medline

Strait DL, Parbery-Clark A, Hittner E, Kraus N (2012) Musical training during early childhood enhances the neural encoding of speech in noise. Brain Lang 123:191-201. CrossRef Medline

Wever EG, Bray CW (1930) The nature of acoustic response: The relation between sound frequency and frequency of impulses in the auditory nerve. Journal of Experimental Psychology 13:373-387. CrossRef

Wong PC, Skoe E, Russo NM, Dees T, Kraus N (2007) Musical experience shapes human brainstem encoding of linguistic pitch patterns. Nat Neurosci 10:420-422. Medline

Wong PC, Warrier CM, Penhune VB, Roy AK, Sadehh A, Parrish TB, Zatorre RJ (2008) Volume of left Heschl's gyrus and linguistic pitch learning. Cereb Cortex 18:828-836. CrossRef Medline

Wozniak JR, Lim KO (2006) Advances in white matter imaging: a review of in vivo magnetic resonance methodologies and their applicability to the study of development and aging. Neurosci Biobehav Rev 30:762-774. CrossRef Medline

Zatorre RJ (1988) Pitch perception of complex tones and human temporallobe function. J Acoust Soc Am 84:566-572. CrossRef Medline

Zatorre RJ, Belin P (2001) Spectral and temporal processing in human auditory cortex. Cereb Cortex 11:946-953. CrossRef Medline

Zatorre RJ, Belin P, Penhune VB (2002) Structure and function of auditory cortex: music and speech. Trends Cogn Sci 6:37-46. CrossRef Medline

Zhu L, Bharadwaj H, Xia J, Shinn-Cunningham B (2013) A comparison of spectral magnitude and phase-locking value analyses of the frequencyfollowing response to complex tones. J Acoust Soc Am 134:384-395. CrossRef Medline 